\title{
A Case of Severe Human Granulocytic Anaplasmosis in an Immunocompromised Pregnant Patient
}

\author{
Marijo Aguilera $^{\mathrm{a}, \mathrm{c}}$, Anne Marie Furuseth ${ }^{\mathrm{b}}$, Lauren Giacobbe ${ }^{\mathrm{a}}$, Katherine Jacobs ${ }^{\mathrm{a}}$, Kirk Ramin ${ }^{\mathrm{a}}$
}

\begin{abstract}
Human granulocytic anaplasmosis (HGA) is a tick-borne disease that can often result in persistent fevers and other non-specific symptoms including myalgias, headache, and malaise. The incidence among endemic areas has been increasing, and clinician recognition of disease symptoms has aided in the correct diagnosis and treatment of patients who have been exposed. While there have been few cases reported of HGA disease during pregnancy, all patients have undergone a relatively mild disease course without complications. HGA may cause more severe disease in the elderly and immunocompromised. Herein, we report an unusual presentation and severe disease complications of HGA in a pregnant female who was concomitantly immunocompromised due to azathioprine treatment of her Crohn's disease. Following successful treatment with rifampin, she subsequently delivered a healthy female infant without any disease sequelae.
\end{abstract}

Keywords: Anaplasma; Ehrlichiosis; Tick-borne disease pregnancy; Rifampin

\section{Introduction}

Human granulocytic anaplasmosis (HGA), reported as human granulocytic ehrlichiosis (HGE) prior to a taxonomic change in 2001, is a zoonotic tick-borne disease caused by Anaplasma phagocytophilum. It was first recognized as a human disease in 1994, and recent estimates of annual incidence approach 1.6 per million in the United States [1]. The clinical presentation is often non-specific and may include fever, chills, malaise, headache, myalgias, nausea, vomiting, and rash. More severe mani-

Manuscript accepted for publication July 10, 2012

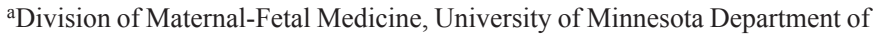
Obstetrics and Gynecology, Minneapolis, MN, USA

bAllina Medical Clinic Obstetrics and Gynecology, Minneapolis, MN, USA ${ }^{\mathrm{c} C}$ Corresponding Author: Marijo N. Aguilera, Division of Maternal-Fetal Medicine, University of Minnesota Department of Obstetrics and Gynecology, 606 24th Avenue South, Suite 401, Minneapolis, MN 55454, USA.

Email:mnaguilera5@hotmail.com

doi: http://dx.doi.org/10.14740/jmc811w festations include respiratory or neurologic involvement, acute renal failure, invasive opportunistic infections and a shocklike illness [2-4]. Recent delineation of the various species associated with ehrlichia infections and an increased understanding of the epidemiology has augmented our knowledge of these tick-borne diseases. However, a detailed understanding of HGA infections in both immunocompromised and pregnant patients is limited. We report a case of a severe HGA infection presenting as an acute exacerbation of Crohn's disease in a pregnant immunocompromised patient.

\section{Case Report}

A 34-year-old primigravida at $17+2$ weeks' gestation presented to the emergency department (ED) in an urban setting of Minneapolis, MN with a 3-day history of fever, chills, headache, nausea, and bilateral flank pain. She had been diagnosed with a urinary tract infection earlier that day at a local urgent care clinic and was started on amoxicillin. Due to persistent pain and a fever of $104.4^{\circ} \mathrm{F}$, she returned to the ED. Her exam was otherwise normal with the exception of bilateral costovertebral angle tenderness. She was afebrile in the ED with a temperature of $99.5^{\circ} \mathrm{F}$, and her other vital signs were within normal limits. Laboratory evaluation including a complete blood count, liver enzymes, and basic metabolic panel did not reveal abnormal findings: white blood cell count of 6,600 cells $/ \mu \mathrm{L}$, hemoglobin level of $12.9 \mathrm{~g} / \mathrm{dL}$, platelet count of 155,000 cells $/ \mu \mathrm{L}$, and creatinine of $0.58 \mathrm{mg} / \mathrm{dL}$. Her urinalysis was not consistent with a urinary tract infection. Further evaluation revealed a history of a recent deer tick bite 4 days prior after attending a camp in a wooded area located in Pine County, Minnesota. A Lyme screen was subsequently performed, and her antibiotics were changed to allow empiric treatment for Lyme disease with amoxicillin and clavulanic acid. She did not have evidence of a rash. Blood and urine cultures were drawn, and she was discharged home after she had improved clinically with fluid hydration in the ED. Two days later she continued to have fevers of $104^{\circ} \mathrm{F}$ despite antibiotics and antipyretics, thus she presented to her obstetrics clinic. Follow-up from her ED visit demonstrated a negative Lyme serology in addition to negative blood and urine cultures. Due to complaints of a sore throat, group A streptococcus and mononucleosis spot tests were drawn and both were negative. A complete blood count, which was repeated to evaluate for 
leukocytosis, revealed thrombocytopenia with a platelet count of 50,000 cells $/ \mu \mathrm{L}$. She did not have any evidence of bleeding, thus she was sent home and instructed to return with any changes in her symptoms.

The following day, she again presented to the ED with persistent fevers, nausea, and headache, but additionally noted new onset abdominal pain, arthralgias, chest pain and respiratory difficulty. She appeared to be in moderate distress. She was tachycardic at 123 beats/min, tachypneic at 22 respirations/min, and slightly hypotensive with a blood pressure of $92 / 55$. She was afebrile with a temperature of $98.3^{\circ} \mathrm{F}$. Her physical exam was unremarkable except for rebound tenderness and voluntary guarding in the right lower quadrant. Laboratory evaluation in the ED was significant for elevated liver enzymes, renal insufficiency, elevated lactate dehydrogenase, and pancytopenia. Laboratory value abnormalities were as follows: white blood cell count 3,600 cells $/ \mu \mathrm{L}$ with a differential count that included $93.8 \%$ neutrophils, $4.1 \%$ lymphocytes, and $1.4 \%$ bands; hemoglobin level $10.6 \mathrm{~g} / \mathrm{dL}$; platelet count 30,000 cells $/ \mu \mathrm{L}$; creatinine $1.64 \mathrm{mg} / \mathrm{dL}$; ALT $31 \mathrm{U} / \mathrm{L}$ (normal range: 0 - $50 \mathrm{U} / \mathrm{L}$ ); AST $140 \mathrm{U} / \mathrm{L}$ (normal range: 0 - $45 \mathrm{U} / \mathrm{L}$ ); total bilirubin $1.7 \mathrm{mg} / \mathrm{dL}$ (normal range: $0.2-1.3 \mathrm{mg} / \mathrm{dL}$ ); lactate dehydrogenase 1,745 U/L (normal range: 325 - $750 \mathrm{U} / \mathrm{L}$ ); lactate $3.5 \mathrm{mmol} / \mathrm{L}$ (normal range: $0.7-2.1 \mathrm{mmol} / \mathrm{L}$ ); and INR 1.31 (normal range: 0.86 - 1.14). An EKG was performed due to her complaints of chest pressure, and it demonstrated sinus tachycardia without other concerning findings. Her chest Xray revealed a small left basilar infiltrate, consistent with probable pneumonia. Her oxygen saturations remained stable with supplemental oxygen as needed. Repeat blood and urine cultures were drawn in addition to Lyme PCR (serum sample) and serology for toxoplasmosis, rubella, herpes simplex virus, and cytomegalovirus. At this point, in light of her Crohn's disease and the possibility for an acute abdominal process, the patient was admitted (at $17+5$ weeks' gestation) for further evaluation. Both surgical and gastroenterology consultations were requested. The gastroenterologist was concerned for a localized Crohn's abscess, thus an MRI was recommended. Ertapenem was started as empiric treatment for a possible intra-abdominal catastrophe and possible pneumonia. Her azathioprine treatment for Crohn's disease was held given the concern for an abscess with sepsis and leukopenia. A diagnostic laparoscopy was considered, but due to her severe thrombocytopenia, it was decided to continue with expectant management. The MRI findings ultimately were not consistent with an acute appendicitis, abscess, or flare of Crohn's disease.

In addition to the aforementioned clinical evaluation, a peripheral blood smear was sent given her history of a recent tick bite and persistent fevers. Later that evening, the pathologist called urgently to report that the preliminary stain had identified the presence of anaplasma species with an abundance of morulae present in granulocytes. An infectious disease consultation was obtained and it was recommended that treatment be initiated promptly. A. phagocytophilim and E. chaffeensis antibody titers were ordered, and she was treated with rifampin $600 \mathrm{mg}$ daily. The previous antibiotics were discontinued.

Over the following 3 - 4 days, the patient began to improve clinically, with complete resolution of her abdominal pain, fever, malaise, and headache. Her renal function was adequate and her creatinine had decreased to $0.94 \mathrm{mg} / \mathrm{dL}$ by hospital day 2 . The platelet count reached a nadir of 23,000 cells $/ \mu \mathrm{L}$ on hospital day 3 and increased steadily thereafter to 58,000 cells $/ \mu \mathrm{L}$ by hospital day 5 . Similarly, the liver enzymes peaked on hospital day 4 (AST: $357 \mathrm{U} / \mathrm{L}$ and ALT: $158 \mathrm{U} / \mathrm{L}$ ) and had decreased substantially by hospital day 5 (AST: 120 U/L and ALT: 176 $\mathrm{U} / \mathrm{L})$. Other laboratory values had also improved on the day of hospital discharge (day 5) as follows: white blood cell count 14,800 cells $/ \mu \mathrm{L}$ (nadir of 2,400 cells $/ \mu \mathrm{L}$ ) with a differential count that included 52\% neutrophils and 40\% lymphocytes; hemoglobin level $11.3 \mathrm{~g} / \mathrm{dL}$; creatinine $0.51 \mathrm{mg} / \mathrm{dL}$; lactate dehydrogenase $994 \mathrm{U} / \mathrm{L}$ (normal range: 325 - $750 \mathrm{U} / \mathrm{L}$ ); and INR 0.93 (normal range: $0.86-1.14$ ). The patient had not experienced any changes in her bowel movements that would further suggest a Crohn's exacerbation, and she had normal bowel function on the day of discharge. Likewise, her chest pain and shortness of breath had resolved. Her A. phagocytophilim and $E$. chaffeensis antibody titers had returned as negative. She completed a 10-day course of rifampin after discharge.

In regards to her pregnancy, a comprehensive ultrasound was performed on hospital day 4 at $18+1$ weeks' gestation. The fetus weighed $284 \mathrm{~g}$, and the amniotic fluid volume was normal with an index of $18 \mathrm{~cm}$. The fetal heart rate was within normal range at 155 beats/min, and all visualized fetal anatomy was normal with the exception of bilateral choroid plexus cysts. Middle cerebral Doppler studies were evaluated for possible fetal anemia given the severe maternal hematologic abnormalities and acute infection, and these were normal. There was no evidence of fetal hydrops or other non-reassuring findings. Follow-up ultrasounds demonstrated resolution of the choroid plexus cysts, appropriate fetal growth, and no other concerning findings. Follow-up A. phagocytophilim and E. chaffeensis antibody titers were performed at 4 months following disease resolution (at 36 weeks' gestation) and were negative. The remainder of her pregnancy was uncomplicated, and she subsequently delivered a healthy female infant weighing 7 pounds, 8 ounces via primary elective cesarean section at 39 weeks' gestation. Apgar scores were 9 and 9 at 1 and 5 min, respectively. Serology for A. phagocytophilim and E. chaffeensis antibody titers was performed on the infant on day 2 of life, and both returned as negative. The infant did not have any other evidence of disease and was discharged to home with her mother on hospital day 4.

\section{Discussion}

Human infection with HGA and disease awareness has increased steadily since the 1990s, and new areas of infection continue to arise internationally. The clinical presentation and course of HGA is generally mild, although hospitalization occurs in $36 \%$ of patients. Of those hospitalized, $7 \%$ are admitted to the ICU due to severe complications such as shock-like syndrome, respiratory and neurological involvement [5-9]. These severe courses are most commonly seen in immunocompromised persons. Since cellular immunity is the key frontline host defense mechanism against rickettsial infections, any impairment in cellular immunity may result in a more severe clinical disease [6-8]. Although the number of HGA cases has 
been increasing, reports of HGA during immunosuppression or pregnancy remain limited.

Among the 11 cases of HGA during pregnancy previously reported, all patients had relatively mild disease without severe complications [2, 9-13]. Muffly reported a case of a first trimester spontaneous abortion after a complicated presumed ehrlichia/anaplasma infection; however, the diagnosis was made on clinical suspicion alone without laboratory confirmation [13]. We thus presume that our case is the first to document a confirmed complicated case of HGA during pregnancy. In addition to the typical symptoms of HGA, our patient presented with acute abdominal pain, chest pain, and respiratory difficulty. She further had anemia, acute renal insufficiency, elevated lactate, mild coagulopathy, and a left basilar infiltrate. Peripheral blood stain examination revealed an abundance of intragranulocytic morulae; however, A. phagocytophilim and $E$. chaffeensis antibody titers remained negative both during acute infection and at 4 months follow-up after disease resolution. We speculate that her immunosuppressed state prevented a robust humoral immune response.

In regards to our choice of treatment, rifampin has been documented for treatment of HGA during pregnancy in six of the 11 prior cases [2, 10-12]. In vitro studies have also shown excellent activity of the rifamycins against Ehrlichia and Anasplasma [4]. She improved rapidly after initiation of rifampin, and did not have any further disease manifestations, medication side effects, or complications with her pregnancy.

Due to a sole report of possible human perinatal transmission by Horowitz et al, we did perform $A$. phagocytophilim and $E$. chaffeensis antibody titers on our patient's infant [9]. These were both negative and the infant had no other disease symptoms. Our case illustrates several new factors that are important in understanding the clinical presentation and treatment of HGA. Importantly, any non-specific febrile illness (and more severe or unusual symptoms in immunocompromised patients) should include tick-borne diseases in the differential diagnosis, even when other factors appear to be of more acute significance. Pregnancy may further complicate treatment, as standard tetracyclines are known to have adverse effects. Rifampin appears to provide adequate coverage for HGA infection during pregnancy, even with severe disease.

\section{Conflict of Interest}

None of the authors have a conflict of interest.

\section{Financial Support}

No financial support was utilized for this study by any of the authors.

\section{References}

1. Ismail N, Bloch $\mathrm{KC}, \mathrm{McBride} \mathrm{JW}$. Human ehrlichiosis and anaplasmosis. Clin Lab Med. 2010;30(1):261-292.

2. Qasba N, Shamshirsaz AA, Feder HM, Campbell WA, Egan JF. A case report of human granulocytic anaplasmosis (ehrlichiosis) in pregnancy and a literature review of tick-borne diseases in the United States during pregnancy. Obstet Gynecol Surv. 2011;66(12):788-796.

3. Demma LJ, Holman RC, McQuiston JH, Krebs JW, Swerdlow DL. Epidemiology of human ehrlichiosis and anaplasmosis in the United States, 2001-2002. Am J Trop Med Hyg. 2005;73(2):400-409.

4. Bakken JS, Dumler JS. Clinical diagnosis and treatment of human granulocytotropic anaplasmosis. Ann N Y Acad Sci. 2006;1078:236-247.

5. Dumler JS. The biological basis of severe outcomes in Anaplasma phagocytophilum infection. FEMS Immunol Med Microbiol. 2012;64(1):13-20.

6. Safdar N, Love RB, Maki DG. Severe Ehrlichia chaffeensis infection in a lung transplant recipient: a review of ehrlichiosis in the immunocompromised patient. Emerg Infect Dis. 2002;8(3):320-323.

7. Whitt SP, Everett ED, Roland W, Dolan S. Ehrlichia chaffeensis--associated cardiomyopathy in a patient with AIDS. Clin Infect Dis. 1999;28(1):140.

8. Martin GS, Christman BW, Standaert SM. Rapidly fatal infection with Ehrlichia chaffeensis. N Engl J Med. 1999;341(10):763-764.

9. Horowitz HW, Kilchevsky E, Haber S, Aguero-Rosenfeld M, Kranwinkel R, James EK, Wong SJ, et al. Perinatal transmission of the agent of human granulocytic ehrlichiosis. N Engl J Med. 1998;339(6):375-378.

10. Buitrago MI, Ijdo JW, Rinaudo P, Simon H, Copel J, Gadbaw J, Heimer R, et al. Human granulocytic ehrlichiosis during pregnancy treated successfully with rifampin. Clin Infect Dis. 1998;27(1):213-215.

11. Casau NC, Hewins ME, Zaleznik DF. Treatment of human granulocytic ehrlichiosis during pregnancy and risk of perinatal transmission. Scand $\mathrm{J}$ Infect Dis. 2002;34(11):853-855.

12. Dhand A, Nadelman RB, Aguero-Rosenfeld M, Haddad FA, Stokes DP, Horowitz HW. Human granulocytic anaplasmosis during pregnancy: case series and literature review. Clin Infect Dis. 2007;45(5):589-593.

13. Muffly T, McCormick TC, Cook C, Wall J. Human granulocytic ehrlichiosis complicating early pregnancy. Infect Dis Obstet Gynecol. 2008;2008:359172. 